\title{
Hierarchical Routing with QoS Constraints in Optical Transport Networks*
}

\author{
Xavier Masip-Bruin, Sergio Sánchez-López, Josep Solé-Pareta \\ Jordi Domingo-Pascual, and Eva Marín-Tordera \\ Departament d'Arquitectura de Computadors, Universitat Politècnica de Catalunya \\ Avgda. Víctor Balaguer, s/n- 08800 Barcelona, Spain \\ \{xmasip, sergio, pareta, jordid, eva\} @ac.upc.es
}

\begin{abstract}
Optical Transport Networks (OTN) with automatical switching capabilities are named ASON. Hierarchical routing is required in the ASON recommendations to achieve scalability. Basically, hierarchical routing consists of three main components, an aggregation scheme, an update policy and a routing algorithm. This paper proposes a new network structure focusing on these three components. We propose a new aggregation scheme; an update policy based on a threshold value; and we also extend an already proposed routing mechanism to be applied to a hierarchical network. Main skill of this routing mechanism is to reduce the connection blocking increase because of selecting paths based on inaccurate routing information. This inaccuracy is introduced both by the aggregation process and by the update policy.
\end{abstract}

Keywords. Hierarchical optical routing, ASON, aggregation schemes, routing inaccuracy.

\section{Introduction}

In recent years the introduction of high capacity and reliable transport networks is being necessary in order to cover the needs of Internet traffic demands. New incoming Internet applications increasingly request greater capacity and guarantees of traffic delivery. Optical Transport Networks (OTN) with automatic switching capabilities (ASON, Automatically Switched Optical Networks) appear as a potential solution to cope with such a situation.

A single carrier optical network may consist of hundreds of network nodes, with each node containing more than one thousand physical ports. An appropriate ASON routing protocol has to be scalable enough for this network size. Some approaches may be used to achieve scalability, such as hierarchical routing, link bundling, and classification of link state information into static and dynamic information. ASON specifications [1] specifically recommend hierarchical routing to guarantee network

\footnotetext{
* This work was partially funded by the MCyT (Spanish Ministry of Science and Technology) under contract FEDER-TIC2002-04344-C02-02 and the CIRIT (Catalan Research Council) under contract 2001-SGR00226.
} 
scalability. The main advantage of hierarchical routing is to reduce large communication overhead while providing efficient routing.

ASON must include a Control Plane able to provide features such as Traffic Engineering. One of the essential components of this Control Plane is the routing protocol, which has to include both a mechanism to disseminate routing information throughout the network and a routing algorithm to select a suitable path. The routing algorithm dynamically computes paths supporting the QoS constraints required by the incoming call. Assuming source-based routing as the most commonly used QoS routing algorithms, routes are computes on the source nodes according to the routing information contained in their network state databases (named Traffic Engineering Database, TED when including QoS parameters). The Control Plane has to provide a flexible, fast and reliable mechanism to both disseminate and update the topology and the available resource information throughout the network. An Optical NNI $(O-N N I)$ should be standardized for ASON to support such a mechanism.

For some time it seemed clear that such an $O-N N I$ would be based on the GMPLS [2] paradigm. Solutions based on the GMPLS paradigm only consider typical Internet protocols such as link state based Interior Gateway Protocols (IGPs). Nevertheless, recently the idea of an $O-N N I$ based on the ATM PNNI [3] paradigm is gaining support. There are some works in the recent literature focusing on adapting the PNNI to the Optical Network Control Plane [4].

The main goal of this paper is to provide ASON with a hierarchical routing in order to ensure the scalability for large worldwide networks. Hierarchical routing functions include two parts:

- Network information dissemination among nodes and networks.

- Constraint based path computation.

According to these main functions we decompose the hierarchical routing problem into three main issues: the aggregation process used to reduce disseminated information throughout the network; the update policy used to keep network state databases perfectly updated; and the lightpath selection process assuming the potential network state inaccuracy introduced by both the aggregation process and the update policy.

The remainder of this paper is organized as follows. Section 2 is devoted to define a hierarchical structure for $A S O N$. In Section 3 we propose a new hierarchical structure including a new aggregation scheme, a new update policy and a new routing mechanism. After that, Section 4 evaluates the proposed mechanism and finally Section 5 concludes the paper.

\section{Hierarchical Routing Issues}

An $A S O N$ should be subdivided into routing areas (RAs), i.e., $\mathrm{X} 1, \mathrm{X} 2, \mathrm{X} 3, \mathrm{Y} 1, \mathrm{Y} 2$ and Y3 (see Fig.1 as an example), which have to contain physical nodes with similar features. The $R A$ nodes should exchange topology and resource information among themselves in order to maintain an identical view of the $R A$. This information should be contained in a Routing Controller $(R C)$ component, which will respond both to requests from connection controllers $(C C)$ for path information needed to set up connections and to requests for topology information from hierarchical mechanisms. 
Each $R A$ should be represented by a "Logical Routing Area Node" (LRAN) in the next hierarchical level. The necessary functions to perform this role should be executed by a node called the "Routing Area Leader" $(R A L)$. This node will receive complete topology state information from all $R A$ nodes and will send information up to the $L R A N$. The propagated information should only include the information needed by the higher level.

As mentioned above, hierarchical routing consists of three main issues: an aggregation process, an update policy and a hierarchical routing algorithm. Next, we shortly introduce these issues.

\subsection{Network Information Aggregation}

There are many possible aggregation schemes. One possible adopted in [3] consists of a complex node representation, which represents the internal structure of each $R A$, called Peer Group, as a symmetric star topology with a uniform radius. B. Awerbuch et al. [5] compared the performance of the different aggregation schemes. They include a star with radius equal to half the cost of the network diameter (DIA), a star radius equal to half the average cost between nodes $(A V E)$, minimum spanning Tree $(M S T)$, Random Spanning Tree (RST) and t-spanner. Other aggregation schemes, namely Full Mesh Aggregation scheme and Asymmetric Simple Aggregation scheme are presented in [6]. They are based on the aggressive mode defined in [3], which chooses the best value of each QoS parameter for every intra-group links. The QoS parameters considered by these schemes are delay, capacity and cost. However, being aware that QoS parameters are different in optical networks and in ATM networks, the QoS parameters considered for optical networks are the propagation delay that is proportional to the fiber distance between two nodes, and the number of wavelengths per link.

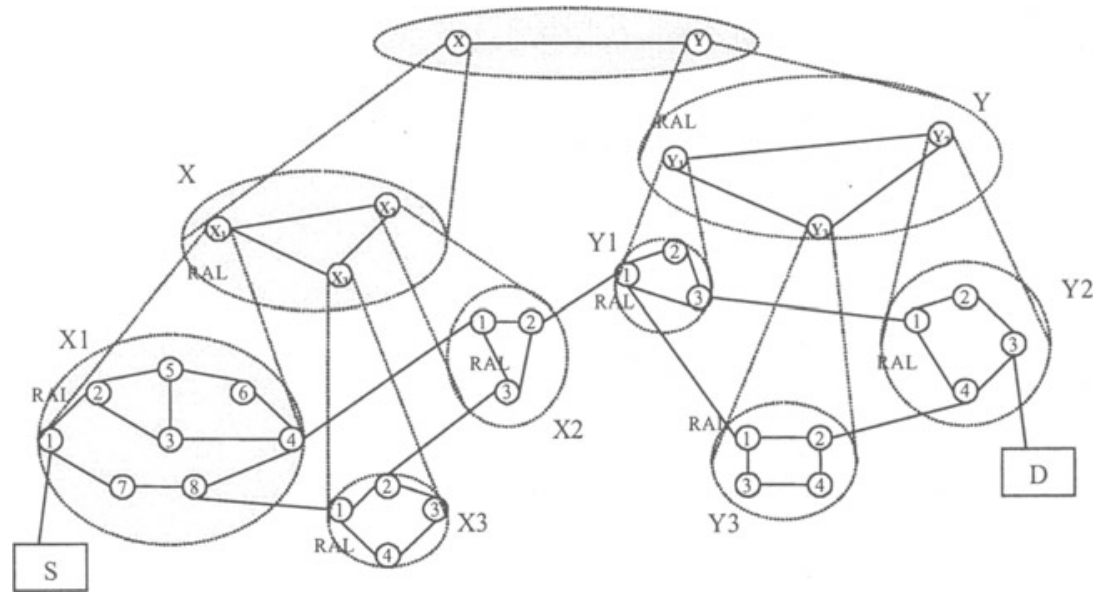

Fig. 1. Hierarchical QoS Routing 


\subsection{Update Policy}

A key factor in the path decision process is the accuracy of the routing information used to compute paths. It has been demonstrated [7] that selecting paths under inaccurate network state information leads to have a significant connection blocking increment, known as the routing inaccuracy problem. There are two main factors in our hierarchical network structure contributing to the inaccuracy: the state aggregation process implemented to reduce the amount of information to be flooded, since information about physical nodes and links is not distributed; and the update policy implemented to reduce the huge number of update messages required to keep TED information perfectly updated. Generally speaking, existing update policies are based on updating by either a periodical refresh or a threshold value. The former is not suitable for large networks mainly owing to network dynamics. The latter is more appropriate since it takes into account the network congestion in the updating decision.

\subsection{Hierarchical Routing Algorithm}

New specific hierarchical routing algorithms should be generated to address the problem of selecting lightpaths under inaccurate network state information. Although the routing inaccuracy problem has been widely analyzed in an IP scenario, there are not many contributions coping with this problem in optical transport network. Main contributions can be found in [7], [8], [9], [10] and [11]. As stated before, in [7] authors only present the effect produced in the blocking probability because of having inaccurate routing information when selecting lightpaths. In [8] the routing inaccuracy problem is addressed by modifying the lightpath control mechanism, and a new distributed lightpath control based on destination routing with rerouting capabilities is suggested. In [9] authors propose a mechanism whose goal is to control the amount of signaling messages flooded throughout the network. The most recent and novel contribution is referred as BYPASS Based Optical Routing mechanism (BBOR) [10], [11]. The $B B O R$ mechanism is a new adaptive source routing mechanism aiming to reduce the connection blocking probability due to perform routing and wavelength assignment decisions under inaccurate network state information. It can also be found in [11] a deeply description of the previously mentioned proposals.

To the authors' best knowledge there is not any proposal in the literature proposing a complete network structure addressing all the three aspects mentioned above and also assuming the routing inaccuracy problem in the lightpath selection process.

\section{A Proposal for Hierarchical Routing in Optical Transport Networks}

This Section proposes a source-based QoS hierarchical routing including a new aggregation scheme to reduce the volume of information to be disseminated throughout the network, an update policy to determine when update messages must be triggered and a routing algorithm to address the routing inaccuracy problem. 


\subsection{The Aggregation Scheme}

Consider a network consisting of $Q$ OXCs. Each node is assumed to have a fixed number of ports. According to the proposed hierarchical structure, an optical network is divided into $M R A s$ connected by border $O X C s$, each one composed of a set of OXCs with similar characteristics. Let $G(Q, U)$ describe the given physical network, where $Q$ is a set of $O X C s$ and $U$ is a set of links (i.e. fibers) connecting the nodes. Let $g(q, u)$ describe the given physical $R A$, where $q$ is the set of nodes $(O X C s)$ in the $R A$ and $u$ is a set of links connecting the nodes within the $R A$. Therefore, $g \in G, q \in Q$ and $u \in U$. Each fiber (link) supports $c$ different wavelengths, i.e. from $\lambda_{1}$ to $\lambda_{\mathrm{c}}$. Moreover, we consider that wavelength conversion does not exist in any OXC. Thus, an incoming call is associated to the same wavelength color along the lightpath.

Generally speaking, aggregation schemes work as follows: Firstly, a $R A$ precomputes all the lightpaths existing between all border nodes along with the QoS parameters allocated to each lightpath. Secondly, an aggregation scheme summarizes this information reducing the amount of data to be flooded throughout the physical network. Finally, the aggregate information from each $R A$ is grouped in a topology database, which will be used by a source node to compute an end-to-end lightpath.

We propose the following network parameters for optical networks:

- $D$ : Propagation delay in a link which is proportional to the fiber distance between two nodes.

- $\quad W_{p}$ : Number of available wavelength of each color in a link

According to the aggregation schemes presented above and the QoS parameters proposed for optical networks, we propose a new aggregation scheme for ASON, namely Node Aggregation Scheme (NAS). The aggregation process performed in this scheme turns out two QoS parameters, the aggregated delay and the aggregated number of available wavelengths.

Node Aggregation Scheme (NAS). Node Aggregation Scheme associates the aggregated QoS parameters to each border node. The process is as follows:

Aggregated Delay $\left(D_{i}\right)$ :

1. Compute all the lightpaths from node $i$ to all border nodes.

2. Add the propagation delay of each link for each lightpath.

3. Select the minimum value among the values computed in the step 2 .

Aggregated number of available wavelength $\left(W_{p}^{i}\right)$ :

1. Compute all the lightpaths from node $i$ to all border nodes.

2. Select the minimum number of wavelength per color that is available on each path.

3. Select the maximum value among the values computed in the step 2 .

Formally, the aggregated delay and available wavelength are defined according to (1) and (2) as follows:

$$
D_{i}=\min _{R_{i j}, \forall j \neq i}\left[\sum_{l \in R_{i j}} D(l)\right],
$$




$$
\underset{p=1 . . c}{W_{p}^{i}}=\max _{R_{i j} ; \forall j \neq i}\left\{\min _{l \in R_{i j}}\left[W_{p}(l)\right]\right\} .
$$

Fig. 3 illustrates the effects of using the proposed aggregation scheme in terms of entries of the Aggregated TED (ATED). It shows a comparison between the ATED size produced when applying the proposed aggregation scheme and the TED size produced when an aggregation scheme is not used. As expected the ATED size is shorter when applying the NAS scheme.

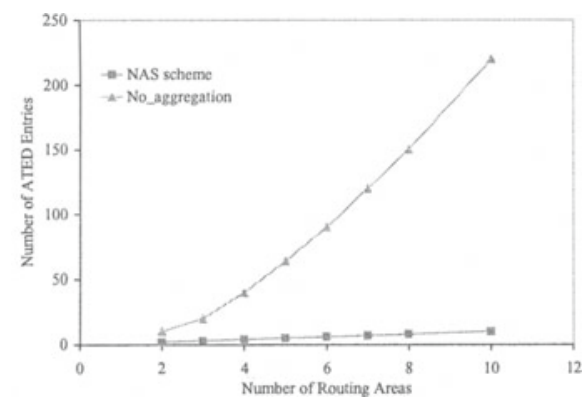

Fig. 3. Comparison with/without aggregation

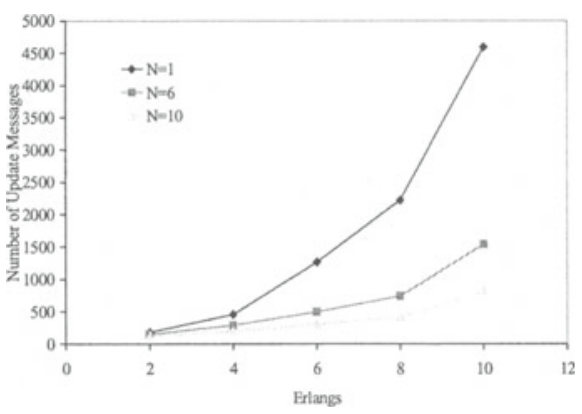

Fig. 4. Number of update messages

\subsection{Update Policy}

The update policy is an important issue of hierarchical routing. Usually, an update policy is implemented by a triggering policy, which decides when update messages must be flooded throughout the network. Most triggering policies are time-based which are not valid for large dynamic networks since they do not represent traffic evolution. Moreover, the accuracy of aggregated information is dependent on the update interval. If the update is set shorter, the aggregate information is more accurate, but the overhead of re-aggregation and information distribution becomes higher. Another triggering policy may be based on sending an update messages whenever there is a change in the network state. This triggering policy guarantees a low inaccuracy but adds an important signaling overhead.

The $B B O R$ mechanism proposed by the authors, introduces a new triggering policy based on a threshold value aiming to include network congestion (available network resources) in the triggering decision. In this way, a network node triggers an update message when a fixed number $N$ of wavelengths changes their status, i.e. after a fixed number of $N$ connections are established or released. This update policy is properly modified to be applied to hierarchical routing. Assuming that the update messages sent by the $R A L$ nodes consist of aggregated information, i.e., information already reduced, the signaling overhead pending to be reduced is that produced into each $R A$. Hence, in hierarchical routing we only apply the update policy into each $R A$.

Fig.4 shows the reduction obtained in the quantity of update messages flooded throughout the hierarchical network topology of Fig.1, when increasing the values of 
$N$. As expected, the larger the $N$ the lower the number of update messages. Note that the case of $N=1$ corresponds to a policy that triggers update messages whenever a change occurs.

\subsection{Routing Algorithm}

ASON recommendations do not specify a routing algorithm in order to compute routing paths. However, it defines a set of features that have to be supported by any routing algorithm running over the optical networks. One of them recommends path computation based on source routing. In this paper we extend the $A L G 3$ [11] routing algorithm inferred from the $B B O R$ to be applied to hierarchical networks. A short description of such an algorithm is now presented.

When a source node is required to establish a new incoming call it selects the kshortest paths to the destination and a free wavelength and sends a set-up message piggybacking the explicit route along the selected lightpath. However, since lightpaths are selected under inaccurate routing information, the selected wavelength may be unavailable at the time of the lightpath set-up, leading to the rejection of the set-up message. The $B B O R$ mechanism addresses this problem by offering an alternative route to all those intermediate nodes that would reject the set-up message. In fact, when an intermediate node detects that the explicitly routed output link has insufficient resources, i.e., there is not the same incoming wavelength to accommodate the new incoming call (no wavelength conversion is provided), it dynamically sends the set-up message along a pre-computed bypass-path which bypasses this link. Therefore, the wavelength availability is the critical parameter in deciding when a lightpath must be rerouted. Assuming that any link is a bundle of $B$ fibers, the main $B B O R$ performance can be detailed according to the next steps:

Defining wavelengths to be bypassed: Those wavelengths in a link that potentially might not be available are defined as Obstruct-Sensitive-Wavelength (OSW). Being $B$ the total number of a certain $\lambda_{i}$ on a link, $R$ the current number of available (not assigned to an already established lightpath) $\lambda_{i}$ on this link we can say that according to the triggering policy defined by the $B B O R$ mechanism, a wavelength $\lambda_{i}$ is defined as OSW, namely $\lambda^{o s}{ }_{i}$ on a certain link, when $R$ is lower or equal than a threshold percentage $T_{p}$ of $N$, being $N$ the number of changes established in the triggering policy to send an update message.

Selecting the lightpath: Once the OSW detection has finished, the lightpath is computed. This process consists of two basic steps, selecting the working path and selecting as many bypass-paths as wavelengths defined as OSWs. On one hand, a new algorithm named ALG3 is proposed when including the OSW in the path selection process. This is done by adding a new parameter named $O S W_{i}(L, F)$ where $L$ is the number of links where $\lambda_{i}$ has been defined as OSW and $F$ is the minimum value of available $\lambda_{i}$ along the lightpath. This is done by adding a new parameter named $O S W_{i}$ $(L, F)$ where $L$ is the number of links where $\lambda_{i}$ has been defined as OSW and $F$ is the minimum value of available $\lambda_{i}$ along the lightpath. Hence, $L$ represents the degree of obstruction and $F$ the degree of congestion of the path. The weight associated to each link is represented by the factor $L / F$. This factor stands for a balance between the number of potentially obstructed links and the real congestion. Moreover, since longer 
paths than the shortest ones can be selected, the length of the path is also included in the path decision. Hence, in order to avoid those paths that are either widest (in terms of wavelength availability) but too long or shortest but too narrow, the weight factor of each path is modeled by $F_{p}$ according to

$$
F_{p}=H\left(\frac{L}{F}\right),
$$

being $H$ the path length. Then, $A L G 3$ selects that $\lambda$ in the pre-selected k-shortest paths minimizing the $F_{p}$ value. On the other hand, once the working path is selected a bypass-path must be computed for those wavelengths defined as $O S W$ in this lightpath. Although other criteria could be used to compute the bypass-paths, such as minimizing the number of wavelengths defined as $O S W$, the shortest (number of hops) bypass-paths are selected. Summarizing, in order to explicitly distribute in the set-up message the bypass-paths, source nodes must perform both the detection of those wavelengths on a link that potentially cannot be available when establishing the path, and the computation of a bypass-path for each one of these wavelengths.

In this paper, $A L G 3$ is modified to be adapted to hierarchical routing turning out a new routing algorithm named $A L G 3 \_H$. In this hierarchical structure, wavelengths can be defined as $O S W$ on different hierarchical levels. This implies two main differences regarding a flat network structure. The former, the $O S W(L, F)$ value can be computed on each hierarchical level. Hence, the expression used so far to model the weight factor $F_{p}$ of each path must be adapted to these different $O S W(L, F)$ values. The new weight factor is $F_{p_{-} H}$ and can be computed according to.

$$
F_{p_{-} H}=\sum_{i=1}^{n} F_{p}^{i},
$$

where $n$ is the number of hierarchical levels and $F_{p}{ }^{i}$ is the parameter defined in (3) per each hierarchical level. The selected wavelength will be that minimizing $F_{p_{-} H^{*}}$

The latter, bypass-paths may be computed on each hierarchical level. Meanwhile the ingress node for each $R A$ must compute the route and the required bypass-paths along its network, the source node receiving the call request must compute the path to the destination node and the required bypass-paths on each hierarchical level.

Bypass-paths usage: Once the working lightpath is computed the set-up message is sent along the selected route. Intermediate nodes can send this set-up message along either the working path, i.e., the selected wavelength is indeed available, or the bypass-path, i.e., the selected wavelength is not really available. It is worth noticing that bypass-paths are only used when the selected wavelength is really not available at the path set-up time in that link defined as $O S W$.

\section{Performance Evaluation}

Before evaluating our proposal, we present an example to illustrate its performance. The example is based on the topology shown in Fig.1. Considering that every $O X C$ includes control functions with signaling capabilities, we assume 10 fibers per link and 4 wavelengths per fiber. Update messages are sent according to $N=6$ and a 
wavelength is defined as $O S W$ according to $T_{p}=50 \%$ (i.e., when the minimum number of available wavelengths on this link is lower than or equal to 3 ). These values are obtained from the simulations performed in [10] .Suppose that incoming call requests arrive between nodes $S$ and $D$. Shaded areas in Fig.1 show the whole network perspective seen by nodes belonging to Routing Area X1. Each node has a topology database containing both complete topology information of the $R A \mathrm{X} 1$ and information summary of the rest of the network. This is represented in Fig. 1 by three different types of links, which are grouped on either physical or logical links. For example, physical link X11-X12 stands for available resources in the link connecting nodes $\mathrm{X} 11$ and X12. The physical link X14-X2 stands for the available resources in the link connecting RA X1 to $R A \mathrm{X} 2$ through node $\mathrm{X} 14$. Finally, logical link $\mathrm{X} 1-\mathrm{X} 2$ stands for the aggregated information of $R A \mathrm{X} 2$ from the node directly connected to $R A \mathrm{X} 1$, i.e., $\mathrm{X} 21$. This aggregated information of the logical link $\mathrm{X} 1-\mathrm{X} 2$ has been obtained according to the information of the topology and available resources database shown in Table 1 and an aggregation process applied to that database, which is described as follows.

Table 1. Topology/Available Resource Database in X2

\begin{tabular}{|l|r|r|r|r|r|}
\hline Link & $\lambda_{1}$ & $\lambda_{2}$ & $\lambda_{3}$ & $\lambda_{4}$ & $\mathrm{D}$ \\
\hline $\mathrm{X} 21-\mathrm{X} 22$ & 2 & 3 & 4 & 7 & 1 \\
\hline $\mathrm{X} 21-\mathrm{X} 23$ & 1 & 5 & 3 & 2 & 1 \\
\hline $\mathrm{X} 23-\mathrm{X} 22$ & 4 & 1 & 6 & 5 & 1 \\
\hline
\end{tabular}

Firstly, the Node Aggregation Scheme (NAS) summarizes the information of Table 1 , according to the expressions (3) and (4). Feasible lightpaths from X21 to X22 and $\mathrm{X} 23$ are: a) X21-X22, b) X21-X23 and c) X21-X23-X22. Therefore, according to (2) the aggregated available wavelength for each color is represented in (5). Moreover, according to (1) the aggregated delay is also represented in (6).

$$
\begin{gathered}
W_{1}^{1}=2 ; W_{2}^{1}=3 ; W_{3}^{1}=4 ; W_{4}^{1}=7, \\
D_{1}=\min _{a, b, c}\left[\sum_{l \in a, b, c} D(l)\right]=\min [1,1,2]=1 .
\end{gathered}
$$

Secondly, X21 bundles its information and disseminates it throughout the $R A$ 's according to a flooding mechanism proposed in [4].

Finally, Table 2 depicts the topology database in X1 built according to the received dissemination messages. In particular, the topology database provides all the information required to compute a route from the given node to any reachable node.

When a call request from node $S$ to node $D$ reaches node $\mathrm{X} 11$, this node applies $A L G 3 \_H$ to select the lightpath based on the information represented in Table 2. Table 3 shows possible shortest paths from node $S$ to node $D$ as well as the $O S W(L, F)$ value on each hierarchical level. 
Table 2. Topology/Available Resource Database in X1

\begin{tabular}{|c|c|c|c|c|c|c|c|c|c|c|c|}
\hline Link & $\lambda_{1}$ & $\lambda_{2}$ & $\lambda_{3}$ & $\lambda_{4}$ & $D$ & Link & $\lambda_{1}$ & $\lambda_{2}$ & $\lambda_{3}$ & $\lambda_{4}$ & $D$ \\
\hline $\mathrm{X} 11-\mathrm{X} 12$ & 6 & 3 & 3 & 6 & 1 & $\mathrm{X} 17-\mathrm{X} 18$ & 0 & 4 & 6 & 1 & 1 \\
\hline $\mathrm{X} 12-\mathrm{X} 13$ & 2 & 3 & 6 & 0 & 1 & $\mathrm{X} 18-\mathrm{X} 14$ & 6 & 6 & 0 & 6 & 1 \\
\hline $\mathrm{X} 13-\mathrm{X} 14$ & 6 & 3 & 0 & 2 & 1 & $\mathrm{X} 14-\mathrm{X} 2$ & 6 & 7 & 7 & 5 & 1 \\
\hline $\mathrm{X} 12-\mathrm{X} 15$ & 6 & 2 & 0 & 1 & 1 & $\mathrm{X} 18-\mathrm{X} 3$ & 5 & 6 & 7 & 5 & 1 \\
\hline $\mathrm{X} 15-\mathrm{X} 13$ & 6 & 6 & 6 & 6 & 1 & $\mathrm{X} 1-\mathrm{X} 2$ & 2 & 3 & 4 & 7 & 1 \\
\hline $\mathrm{X} 15-\mathrm{X} 16$ & 0 & 7 & 3 & 3 & 1 & $\mathrm{X} 1-\mathrm{X} 3$ & 1 & 2 & 3 & 5 & 1 \\
\hline $\mathrm{X} 16-\mathrm{X} 14$ & 1 & 1 & 1 & 1 & 1 & $\mathrm{X} 3-\mathrm{X} 2$ & 4 & 4 & 5 & 7 & 3 \\
\hline $\mathrm{X} 11-\mathrm{X} 17$ & 6 & 3 & 1 & 6 & 1 & $\mathrm{X}-\mathrm{Y}$ & 4 & 3 & 2 & 3 & 5 \\
\hline
\end{tabular}

Table 3. Routing Table in X11

\begin{tabular}{|c|cccc|c|c|}
\hline Route I & $\lambda_{1}$ & $\lambda_{2}$ & $\lambda_{3}$ & $\lambda_{4}$ & $D$ & $O S W(L, F)$ \\
\hline $\mathrm{X} 11-\mathrm{X} 12-\mathrm{X} 13-\mathrm{X} 14-\mathrm{X} 2$ & 2 & 3 & 0 & 0 & 4 & $\lambda_{1}(1,2), \lambda_{2}(3,3)$ \\
\hline $\mathrm{X} 1-\mathrm{X} 2$ & 2 & 3 & 4 & 7 & 1 & $\lambda_{1}(1,2), \lambda_{2}(1,3)$ \\
\hline $\mathrm{X}-\mathrm{Y}$ & 4 & 3 & 2 & 3 & 5 & $\lambda_{2}(1,3), \lambda_{3}(1,2), \lambda_{4}(1,3)$ \\
\hline Route 2 & $\lambda_{1}$ & $\lambda_{2}$ & $\lambda_{3}$ & $\lambda_{4}$ & $D$ & $O S W(L, F)$ \\
\hline $\mathrm{X} 11-\mathrm{X} 17-\mathrm{X} 18-\mathrm{X} 3$ & 0 & 3 & 1 & 1 & 3 & $\lambda_{2}(1,3), \lambda_{3}(1,1), \lambda_{4}(1,1)$ \\
\hline $\mathrm{X} 1-\mathrm{X} 3-\mathrm{X} 2$ & 1 & 2 & 3 & 5 & 4 & $\lambda_{1}(1,1), \lambda_{2}(1,2), \lambda_{3}(1,3)$ \\
\hline $\mathrm{X}-\mathrm{Y}$ & 4 & 3 & 2 & 3 & 5 & $\lambda_{2}(1,3), \lambda_{3}(1,2), \lambda_{4}(1,3)$ \\
\hline
\end{tabular}

Table 4. $\mathrm{F}_{\mathrm{p}-\mathrm{H}}$ value for Route 1 and Route 2

\begin{tabular}{|l|c|c|l|l|c|c|}
\hline \multicolumn{1}{|c|}{ Route 1 } & $F_{p}\left(\lambda_{1}\right)$ & $F_{p}\left(\lambda_{2}\right)$ & Route 2 & $F_{p}\left(\lambda_{2}\right)$ & $F_{p}\left(\lambda_{3}\right)$ & $F_{p}\left(\lambda_{4}\right)$ \\
\hline $\mathrm{X} 11-\mathrm{X} 12-\mathrm{X} 13-\mathrm{X} 14-\mathrm{X} 2$ & 2 & 4 & $\mathrm{X} 11-\mathrm{X} 17-\mathrm{X} 18-\mathrm{X} 3$ & 1 & 3 & 3 \\
\hline $\mathrm{X} 1-\mathrm{X} 2$ & 0.5 & 0.33 & $\mathrm{X} 1-\mathrm{X} 3-\mathrm{X} 2$ & 2 & 0.67 & 0 \\
\hline $\mathrm{X}-\mathrm{Y}$ & & 1.67 & $\mathrm{X}-\mathrm{Y}$ & 1.67 & 2.5 & 1.67 \\
\hline \multicolumn{1}{|c|}{} & 2.5 & 6 & $F_{p-H}$ & 4.67 & 6.17 & 4.67 \\
\hline
\end{tabular}

Once shortest paths are selected and the $O S W(L, F)$ has been defined, $A L G 3$ must select the wavelength to be used. This is performed by computing the $F_{p_{-} H}$ value for each wavelength on both paths according to (4). Table 4 illustrates the $F_{p_{-} H}$ computation. $A L G 3$ selects that wavelength minimizing the $F_{p_{-} H}$ value. Therefore, the first route is selected and $\lambda_{1}$ will be used to transport the traffic.

Once the lightpath is selected node X11 must select the required bypass-paths. The selected wavelength $\lambda_{1}$ has been defined as $O S W$ in two different levels therefore two bypass-paths must be computed. On the one hand, in the low level $\lambda_{1}$ is OSW in the link X12-X13. A bypass-path across X15, i.e. X12-X15-X13, may be used to bypass the link where the selected wavelength has been defined as OSW. On the other hand, $\lambda_{1}$ has also been defined as $O S W$ in the logical link X1-X2. This means that logical node $\mathrm{X} 1$ should compute a bypass-path to bypass this link. There is only one option to bypass the logical link X1-X2, that made up of X1-X3-X2. The usage of this bypass-path must be carefully analyzed. It must be noticed that the edge node from $\mathrm{X} 1$ to $\mathrm{X} 2$ is different from that connecting $\mathrm{X} 1$ to $\mathrm{X} 3$. In fact, $\mathrm{X} 14$ and $\mathrm{X} 18$ connects $\mathrm{X} 1$ to $\mathrm{X} 2$ and to $\mathrm{X} 3$ respectively. Assuming that the primary path ends at $\mathrm{X} 14$, the bypass-path to $\mathrm{X} 2$ is made up of $\mathrm{X} 14-\mathrm{X} 18-\mathrm{X} 3-\mathrm{X} 2$. When node $\mathrm{X} 14$ receives the set-up message it forwards this message to $\mathrm{X} 2$ through $\mathrm{X} 14-\mathrm{X} 2$ if the selected wavelength is 
available on this link. At this point the set-up message reaches $R A \mathrm{X} 2$ through $\mathrm{X} 21$. If the selected wavelength is not available on the logical link X1-X2, node X21 must send the set-up message back to find the computed bypass-path. A crankback mechanism is implemented to send the message back to node X14. Unlike the traditional crankback mechanism where the alternative route is computed in all nodes in the reverse path, in our proposal the route must not be computed instead only first node of the bypass-path must be reached.

Once the proposed hierarchical network structure has been analyzed by the illustrative example presented above, we also use the network topology shown in Fig. 1 to evaluate our proposal. However, unlike the illustrative example, in this case we suppose a 5-fiber topology, with 16 wavelengths on all the fibers on all the bi-directional links. Call arrivals are modeled by a Poisson distribution and the connection holding time is assumed to be exponentially distributed.

In Fig. 5 the impact on the blocking probability reduction obtained when applying the aggregation schemes and the update policy proposed in this paper is clearly presented. A value of $N$ must be selected to implement the update policy. According to the results obtained in Fig. 4 and considering that the larger the $N$ the lower the number of update messages but the larger the computational cost of the BBOR mechanism, a value of 6 is assumed for the $N$. Moreover, a value of the $T_{p}$ parameter must also be considered. Again, $T_{p}=50 \%$ to reduce the cost of the $B B O R$ mechanism. A detailed impact of the $N$ and the $T_{p}$ values on global network performance can be found in [10]. The routing algorithm used to select the routes is based on applying the First-Fit (FF) heuristic [12] on the pre-computed shortest path. Analyzing the results obtained in Fig. 5 we can observe that compared to the case when there is not information aggregation only a minor blocking probability increase of $3.5 \%$ is obtained by the NAS scheme.

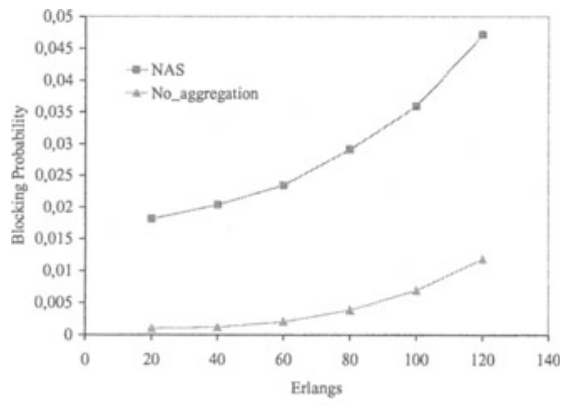

Fig. 5. Blocking probability with/without aggregation

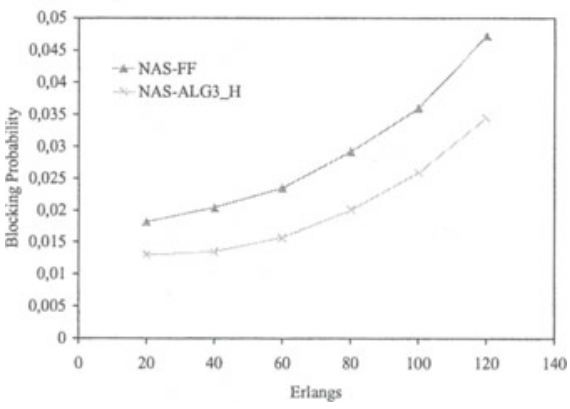

Fig. 6. Blocking probability for the First-Fit and the ALG3_H

In Fig. 6 we evaluate the impact on the blocking probability because of applying the $A L G 3 \_H$ inferred from the $B B O R$ mechanism. In fact, NAS scheme performs better when the $A L G 3 \_H$ is also included. Compared to the First-Fit heuristic a reduction of $1.25 \%$ is obtained in the blocking probability under the higher network load by the NAS scheme. 


\section{Conclusions}

In this paper a new source-based hierarchical routing mechanism is proposed to be applied to optical networks. The proposed routing mechanism deals with three main aspects related to hierarchical routing, namely the aggregation schemes, the update policies and the routing algorithms. We propose a new aggregation scheme to reduce the overhead produced by the routing information dissemination in large networks. We also propose an update policy to reduce the number of update messages needed to guarantee accurate network state information in the border nodes that select paths. Finally, a new routing algorithm is also presented to address the effects on global network performance because of having a hierarchical structure. Mainly this routing algorithm is oriented to reduce the negative effects on the blocking performance because of selecting paths under inaccurate network state information.

The source-based hierarchical routing proposed in this paper has been evaluated on a realistic network topology by simulation. Obtained results show a reduction in the required size of the TED (because of applying the suggested aggregation scheme), a reduction in the update messages flooded throughout the network (because of applying the suggested update policy) and a reduction on the blocking probability (because of applying the suggested routing algorithm).

\section{References}

[1] ITU-T Rec. G8080/Y.1304, "Architecture for the Automatically Switched Optical Network (ASON)", November 2001.

[2] P.Ashwood, et al., "Generalised MPLS - Signalling Functional description", draft-ietfmpls-generalised-signalling-09.txt, February 2003.

[3] ATM Forum, "Private Network to Network Interface Specification Version 1.1", af-pnni0055.002, April 2002.

[4] S. Sánchez-López, J. Solé-Pareta, J. Comellas, J. Soldatos, G. Kylafas, M. Jaeger, "PNNI Based Control Plane for Automatic Switched Optical Networks", Accepted for publication to IEEE Journal of Lightwave Technology, Special Issue on Optical Networks, vol.21, $\mathrm{n}^{\circ} .11$, November 2003.

[5] Awerbuch B., et al., "Routing Through Network with Hierarchical Topology Aggregation", IEEE Symposium on Computer and Communications 1998.

[6] Ben-Jye, et al., "Dynamic Update of Aggregated Routing Information for Hierarchical QoS Routing in ATM Network", ICPADS 2001.

[7] J.Zhou, X.Yuan, "A Study of Dynamic Routing and Wavelength Assignment with Imprecise Network State Information", in Proceedings of ICPP Workshop on Optical Networks, Canada, August 2002

[8] J.Zheng, H.T.Mouftah, "Distributed lightpath control based on destination routing in wavelength-routed WDM networks", Optical Networks Magazine, Vol.3, n:4, pp.38-46, July/August 2002.

[9] S. Darisala, A. Fumagalli, P. Kothandaraman, M. Tacca, L. Valcarenghi, "On the Convergence of the Link-State Advertisement Protocol in Survivable WDM Mesh Networks", in Proceedings of $7^{\text {th }}$ IFIP Working Conference on Optical Network Design \& Modelling, ONDM'03, pp. 433-447, Budapest, Hungary, February 2003. 
[10] X. Masip-Bruin, R. Muñoz, S. Sànchez-López, J. Solé-Pareta, J. Domingo-Pascual, G. Junyent, "An Adaptive Routing Mechanism for Reducing the Routing Inaccuracy Effects in an ASON", in Proc. ONDM'03, pp.333-349, Budapest, Hungary, February 2003.

[11] X. Masip-Bruin, S. Sànchez-López, J. Solé-Pareta, J.Domingo-Pascual, D. Colle, "Routing and Wavelength Assignment under Inaccurate Routing Information in Networks with Sparse and Limited Wavelength Conversion", in Proc. IEEE GLOBECOM 2003, San Francisco, USA, December 2003.

[12] H. Zang, J.P. Jue, B. Mukherjee, "A Review of Routing and Wavelength Assignment Approaches for Wavelength-Routed Optical WDM Networks", Optical Networks Magazine, January 2000. 